All these results indicate that chinoform cannot be metabolized by microsomal electron transfer system, but decreases the components of the electron transfer system. Accordingly, main mechanism of detoxication of chinoform might be ascribed to its esterification in the liver, and chinoform, if it is not esterified, could be toxic or harmful to biological systems such as microsomal electron transfer system.

Zusammenfassung. Nachweis, dass Chinoform, 5-chloro 7-iodo 8-quinolinol, mit Mikrosomen der Rattenleber weder hydroxyliert noch dehalogeniert werden kann. Inkubation mit Chinoform vermindert die Komponenten des elektronischen Transfersystems, was auch mit in vivo-Versuchen festgestellt wurde.

\section{K. Yagi, N. Ohishi, A. TAKaI and S. HatTori}

Institute of Biochemistry, Faculty of Medicine, University of Nagoya, Nagoya (Japan), 3 April 1974.

\title{
Die Wirkung des Glyzins auf chronischen Morphinismus
}

Vor kurzem konnten wir zeigen, dass die synthetische "Substanz P» (SSP) Glyzin (G) im ZNS erhöht". Wir konnten ebenso zeigen, dass SSP die Abstinenzsymptome chronisch morphinisierter Mäuse (CHMM) zurückdrängt ${ }^{2}$. Dieser Befund kompliziert sich durch die Tatsache, dass Nalorphin, welches die Abstinenzsymptome hervorruft, ebenso G im ZNS erhöht ${ }^{3}$. Wir haben in einer früheren Arbeit erwähnt, dass $G$, für welches wir zeigen konnten, dass es die hemoenzephale Barriere durchdrängt ${ }^{4}$, die Abstinenzsymptome der CHMM nicht potenziert ${ }^{3}$. Aus diesen Gründen glauben wir, dass es interessant wäre, die Wirkung des $G$ auf die Abstinenzsymptome CHMM im eventuellen entgegengesetzten Effekt, d.h. im eventuellen Zurückdrängen der Abstinenzsymptome, zu prüfen. Im Versuch waren weisse Mäuse von $20 \pm 2 \mathrm{~g}$ (eigene Zucht); chronischen Morphinismus haben wir nach der Methode Huidobro und Miranda ${ }^{5}$ durch Tablettenimplantation (s.c. $70 \mathrm{mg}$ Morphiumbase) hervorgerufen. Die Tabletten wurden nach der Beschreibung von WAY et al. ${ }^{6}$ hergestellt. Am 4. Tag bekamen die Mäuse 200 und $400 \mathrm{mg} / \mathrm{kg}$ Gryzin i.p. und 30 min nach Glyzin Nalorphin-Bromid $100 \mathrm{mg} / \mathrm{kg}$ i.p. Die Abstinenzsymptome, die sich als Springen und Unruhe manifestierten, wurden in den folgenden 10 min gezählt.

Wie aus der Tabelle ersichtlich, werden bei CHMM die Abstinenzsymptome durch $\mathrm{G}$ unterdrückt. Darnach wirken SSP und $G$ in diesem Experiment synergistisch. Sie unterdrücken Abstinenzsymptome; Nalorphin, welches

\begin{tabular}{lclll}
\hline Gruppe & $\begin{array}{l}\text { Anzahl } \\
\text { Tiere }\end{array}$ & $\begin{array}{l}\text { Dosis } \\
(\mathrm{mg} / \mathrm{kg})\end{array}$ & $\begin{array}{l}\text { Sprungzahl innerhalb } \\
\text { von 10 min }\end{array}$ & $p$ \\
\hline Kontrolien & 6 & - & $38,6 \pm 2,9$ & $<0,01$ \\
Versuchstiere & 10 & 400 & $21,3 \pm 2,5$ & \\
Kontrolien & 6 & - & $66,5 \pm 6,2$ & 0,05 \\
Versuchstiere & 6 & 200 & $42,5 \pm 3,5$ & \\
\hline
\end{tabular}

ebenso G im ZNS erhöht, wirkt umgekehrt. Wir konnten schon früher zeigen, dass $G$ auch auf andere Erregungszustände beruhigend wirkt, z.B. psychomotorische Unruhe, hervorgerufen durch $\beta$ - $\beta$-iminodipropionitril, oder auch Aggressivität der männlichen isolierten Mäuse ${ }^{7}$. Es ist interessant, zu erwähnen, dass Nalorphin die durch $\beta$ - $\beta$-iminodipropionitril erzeugte psychomotorische Unruhe inhibiert, während dieselbe Substanz ohne Wirkung auf die Aggressivität der männlichen isolierten Mäuse ist. Mephenesin das ebenso $G$ im ZNS erhöht, unterdrïckt die Abstinenzsymptome CHMM und die Aggressivität der isolierten aggressiven Mäuse ${ }^{7}$. SSP beruhigt diese Art von Aggressivität ${ }^{2}$. Diese Versuche zeigen, dass SSP und G in einigen Fällen dieselbe Wirkung auf Erregungszustände haben. Es stellt sich die wichtige Frage, ob G auch bei chronischem Morphinismus bei Menschen die Abstinenzsymptome unterdrückt, was von grossem Nutzen sein könnte. Entsprechende Versuche sind im Gange.

Summary. Glycine inhibits withdrawal induced by nalorphine-like synthetic 'substance $\mathrm{P}$ ' on mice chronically treated with morphine.

P. Stern und M. Stern

Pharmakologisches Institut der Medizinischen

Fakultät, YU-71001 Savajevo (Jugoslawien), 2. Juli 1974.

\section{The Effects of a Novel Insecticide on Insect Cuticle}

A new insecticide under development by PhilipsDuphar, PH 60-40, interferes with the development of insect cuticle such that the insect has difficulty in moulting, and dies because the new cuticle ruptures before hardening. Some evidence ${ }^{1,2}$ suggests that this is due to the inhibition of chitin incorporation, thus weakening the cuticle. There is no published information on what happens to the protein fraction in the cuticle of 